\title{
Philosophiques
}

\section{Intentionnalité et référence directe}

\section{Richard Vallée}

Volume 34, numéro 1, printemps 2007

Cosmopolitisme et particularisme

URI : https://id.erudit.org/iderudit/015869ar

DOI : https://doi.org/10.7202/015869ar

Aller au sommaire du numéro

Éditeur(s)

Société de philosophie du Québec

ISSN

0316-2923 (imprimé)

1492-1391 (numérique)

Découvrir la revue

Citer ce document

Vallée, R. (2007). Intentionnalité et référence directe. Philosophiques, 34(1), 159-164. https://doi.org/10.7202/015869ar d'utilisation que vous pouvez consulter en ligne.

https://apropos.erudit.org/fr/usagers/politique-dutilisation/ 


\title{
Intentionnalité et référence directe
}

\author{
RICHARD VALLÉE \\ Université de Moncton \\ RVALLEE@umcs.ca
}

Le livre de Pierre Jacob est une excellente introduction à un grand nombre de problèmes qui ont préoccupé les philosophes analytiques au cours du siècle dernier. Je veux concentrer mon attention sur les termes directement référentiels discutés au chapitre 4. L'intérêt pour ces termes semble difficilement justifiable aux yeux du non initié. Pourquoi passer tant de temps sur les noms propres et les pronoms? Brentano, qui fait l'objet du chapitre 2, définit le mental, ou l'esprit, en disant que tous les phénomènes mentaux ont une intentionnalité, et Pierre précise que l'intentionnalité est cette capacité de représenter un objet qui peut ne pas exister. L'esprit aurait une relation aux objets par le biais de représentations résultant de cette capacité. Cette thèse, sans précisions supplémentaires, surtout en ce qui concerne "représenter ", est suffisamment vague pour qu'il soit difficile de dire ce qui la rendrait fausse sans se faire accuser de l'interpréter de façon peu charitable. L'examen des indexicaux et des noms propres suggère que les notions d'intentionnalité et de représentation mentale trouvent peut-être leurs limites quand elles affrontent ces termes, et que le thème de la relation entre une expression linguistique, une pensée et une chose est plus complexe que ce que l'on croit généralement. Je me pencherai en particulier sur les cas où l'esprit ne peut penser à un objet si cet objet n'existe pas : soi. Ce qui nous ramène au premier chapitre de L'intentionnalité, chapitre consacré à Descartes. La théorie de la référence directe a un caractère plus révolutionnaire que ne le laisse entendre Pierre et laisse croire que nous pensons à certains objets sans l'intermédiaire de représentations mentales de ces objets. Comme il se doit dans le cadre d'une disputatio, je ferai part de mon désaccord avec Pierre en ce qui concerne certains détails de la théorie de la référence directe. Je me permettrai aussi de clarifier un point soulevé par l'auteur, à savoir que la théorie de la référence directe «a pour ambition de conférer aux propositions singulières un rôle central dans l'analyse de la pensée humaine » (p. 87). Les propositions singulières remettent en fait en question notre façon d'analyser la pensée humaine.

Selon une thèse très connue,

A. - Si un individu pense à un objet, alors il a une représentation mentale de cet objet, et il pense à cet objet en vertu de cette représentation mentale.

et

B. - Si un individu a une représentation mentale d'un objet, alors il pense à cet objet en vertu de cette représentation mentale. 
La théorie de la référence directe donne à penser que $\mathrm{A}$ est fausse. Je ne considérerai pas B ici. Supposons que je pense que je suis malade ou que Stumpf est un philosophe. Je pense à moi et à Stumpf. Mais ai-je une représentation de moi ? Ai-je une représentation de Stumpf? Je soutiendrai que l'indexical « je » n'implique pas que j'ai une représentation mentale de moi-même et que les noms propres n'impliquent pas une représentation mentale d'un objet. Pierre montre comment le théoricien de la référence directe, devant certaines énigmes soulevées par les noms propres dans une interprétation de dicto, peut se prévaloir de la notion de mode de présentation. Je crois cependant que ces moyens sous-estiment l'influence des théories de la référence directe sur notre conception de la relation entre les mots, les pensées et les choses.

1. Les noms propres occupent une place importante dans la discussion de la question de savoir comment on peut parler d'un objet qui n'existe pas. Les noms qui occupent l'avant-scène sont bien sûr des noms d'objets de fiction: "Zeus ", "Sherlock Holmes ", etc. Pierre présente la théorie de la référence directe par le biais des noms propres conçus en termes de désignateur rigide de jure ${ }^{1}$. Une description définie comme « le satellite naturel de la Terre » est un terme qui peut être analysé «à la Russell », à l'aide de quantificateurs, de variables, de connecteurs et de prédicats. Notre description aurait la forme « (马 $\mathrm{x})$ satellite de la Terre $\mathrm{x}$. (y) satellite de la Terre y $(\mathrm{y}=\mathrm{x}$ ». Un unique objet, celui qui satisfait la propriété fournie par la description définie, rend vraie une phrase contenant cette description. Une description définie n'est pas un terme référentiel et précise uniquement qu'il existe un et un seul objet qui satisfait la description. Un désignateur rigide de jure: i) désigne un objet auquel il a été assigné; ii) désigne sans l'intermédiaire d'une description de cet objet; et iii) désigne le même objet dans tous les mondes possibles où cet objet existe. Pierre souligne qu' « elle [la théorie de la référence directe] plonge ses racines dans la sémantique de la logique modale quantifiée ». Si la théorie de la référence directe émanant de la théorie des noms propres a sa source, historiquement, dans des problèmes liés à des discussions de logique modale, Barcan Marcus introduisait les noms simplement comme des termes qui sont insensibles aux modalités ${ }^{3}$. Kripke utilise la logique modale pour soutenir que les noms propres n'ont pas le sens de descriptions définies, ou ne sont pas des descriptions définies abrégées, et en arrive à la même conclusion que Barcan Marcus. Les modalités ont une fonction négative: soutenir que les noms ne peuvent être analysés à la Frege ou à la Russell. Donnellan ${ }^{4}$ introduit d'ailleurs les noms comme termes directement référentiels sans utiliser la machinerie de la logique modale. Les noms propres, dans la théorie de la référence directe,

1. S. Kripke, Naming and Necessity, Oxford, Blackwell, 1972.

2. Barcan R., Marcus, «" Modalities and Intensional Languages», Synthese 12, 4, 1961, 303-322.

3. C'est essentiellement la caractérisation des désignateurs rigides qu'on lit p. 90.

4. K. Donnellan, « Proper Names and Identifying Descriptions», Synthese 21, 1970, 335-358. 
sont caractérisés par le fait d'être liés à un objet par assignation, et ils sont directement référentiels parce que cet objet rend vraie la proposition exprimée : il fait partie des conditions de vérité de la phrase. Le critère de rigidité, qui est un critère modal, utilisé afin de caractériser les noms propres comme termes directement référentiels de désignateurs rigides, est accessoire. L'idée est que l'on peut prendre la proposition singulière en question, et ensuite l'évaluer dans différents mondes. En ce sens, on peut éloigner les noms propres comme termes directement référentiels de leur lieu de naissance théorique afin d'en donner la sémantique.

Dans ce contexte, "Stendhal est l'auteur de La Chartreuse de Parme » et «Henri Beyle est l'auteur de La Chartreuse de Parme» expriment la même proposition singulière 5 . Si on peut penser à un objet en utilisant un nom propre, alors on peut penser à un objet sans l'intermédiaire d'une ou de plusieurs propriétés (Russell), ou d'un mode de présentation (sens frégéen) conventionnellement associé au nom, puisque les noms propres « dirigent » la pensée vers cet objet. Supposons que je dise que Stumpf était philosophe. Je pense à Stumpf, et le nom utilisé réfère à un individu, Stumpf, mais n'est pas sémantiquement lié à une manière de penser à Stumpf au sens où cette manière le "viserait ", pour employer la métaphore de Brentano. Ma pensée concerne Stumpf parce que j'utilise ce nom, qui lui a été assigné, et non parce que j'ai un mode de présentation ou une représentation mentale qui le « vise». Le nom ne peut constituer une représentation au sens de Brentano, sinon au risque de devenir une entité mentale. On peut bien sûr dire que le nom représente l'individu, mais en un sens trivial de "représentation ». Si l'on dit que j'ai une fiche ou un dossier ${ }^{6}$ contenant des informations à propos de Stumpf, il est douteux que l'on veuille qualifier cette fiche de représentation mentale grâce à laquelle je pense à Stumpf. Je penserais aussi à Stumpf à cause du nom, et non de la fiche: la fiche concerne l'individu à cause du nom, et le nom ne concerne pas cet individu à cause de la fiche. Supposons maintenant que vous disiez « Richard pense que Stumpf était un philosophe. » Un autre nom coréférentiel avec «Stumpf » pourrait être substitué à "Stumpf » et, selon la théorie de la référence directe, nous aurions un énoncé vrai. $\mathrm{Ni}$ «Stumpf » ni le nom qu'on lui substituerait n'équivaut à une manière de penser à Stumpf. Barcan Marcus, initialement, et Donnellan n'ont pas porté une attention particulière aux noms propres enchâssés dans des termes d'attitude propositionnelle. Kripke l'a fait et, dans «A Puzzle about Belief " ${ }^{7}$, il souligne que le problème qui l'intéresse concerne uniquement l'interprétation de dicto de l'occurrence d'un nom propre (Kripke, 1979, note 7). Il s'agit de cas où il y a échec de la substitution salva veritate de termes co-référentiels dans un contexte d'attitude propositionnelle. Cette

5. On devrait substituer «singulière " à "générale ", à la page 100, lignes 6 et 7.

6. J. Perry, Reference and Reflexivity, CSLI Publications, 2001.

7. S. Kripke, "A Puzzle About Belief », dans A. Margalit (dir. publ.), Meaning and Use, Dordrecht, Reidel, 1979. 
préoccupation ne l'engage pas à se prononcer sur la raison pour laquelle il y a échec dans ce cas précis, ne l'engage pas à affirmer qu'il y a ou non un mode de présentation associé au nom et/ou à l'objet. De fait, il reste muet à ce sujet. Pierre (p. 103) semble identifier une interprétation de re de l'occurrence d'un nom propre à la portée large du terme quantifié, et une occurrence de dicto de ce nom à la portée étroite d'un terme quantifié. Les catégories de re / de dicto valent pour les termes référentiels - parce qu'elles sont définies en termes de substitution de termes coréférentiels -, et les catégories portée large / portée étroite valent pour les termes quantifiés - parce qu'elles sont définies en terme de portée du quantificateur. On doit donc clairement les distinguer. Une proposition singulière ne peut constituer une pensée, parce qu'un locuteur qui utilise "Henri Beyle est l'auteur de La chartreuse de Parme» et un autre qui utilise "Stendhal est l'auteur de La chartreuse de Parme » exprimeraient la même pensée. Mais on peut nier la première phrase et accepter la seconde sans être irrationnel. Comme le souligne Pierre dans son livre, de nombreux néo-russelliens ont supposé qu'il existait un mode de présentation lié au nom et ont posé qu'un énoncé comme "Anne croit que Henri Beyle est l'auteur de La chartreuse de Parme » exprime une proposition qui peut être représentée par:

(马 m) [Croit (Anne < Henri Beyle, auteur de La chartreuse de Parme >, m)]

Je me distance de la position de Pierre selon laquelle «la théorie de la référence directe expulse donc le sens du nom propre en dehors de la proposition explicitement exprimée et l'introduit dans les propositions implicitement communiquées par l'énoncé » (p. 102). Les sens dont il est question sont des sens frégéens, et la thèse de la référence directe est que les noms propres n'ont pas de sens frégéens. On ne fait donc pas que les expulser en dehors de la proposition explicitement exprimée, puisqu'on pose que les noms propres en sont privés. Les modes de présentation, introduits par les néo-russelliens, ne sont pas des sens frégéens. De plus, on ne les caractérise que par leur fonction, et on ne leur assigne aucune autre fonction que d'expliquer l'échec de substitution de noms propres coréférentiels en contexte d'attitude propositionnelle. En cela leur introduction est ad hoc. Je ne crois pas non plus qu'il y ait un argument plausible pour soutenir l'existence d'une telle quantification, sauf un argument du genre: "On doit expliquer l'échec de la substitution de termes coréférentiels, et quoi d'autre utiliser ? » À ces deux titres, la forme de la proposition demeure bien insatisfaisante. Si ce que j'ai dit des noms propres est juste, les noms propres, y compris les noms de fiction, ne sont pas accompagnés d'une représentation mentale de ce à quoi ils réfèrent, et donc la question de savoir comment représenter quelque chose qui n'existe pas ne se pose ni pour les noms propres ni pour les pensées exprimées par des phrases contenant des noms propres. Il n'y a pas de représentation. Peut-être que les noms sont des représentations en un sens trivial. Si l'on veut dire qu'ils résultent de l'intentionnalité du mental, on devrait montrer que des intentions - et, 
comme l'explique clairement Pierre, il faut distinguer intentions et intentionnalité - ne suffisent pas à expliquer leur présence dans les idiolectes. Je conviens que ma position n'aborde pas la question des noms privés de référent. Mais si ce que je dis est vrai, cette question ne relève pas de l'intentionnalité et n'est pas solutionnée en faisant appel à cette notion.

2. Les indexicaux, qui font l'objet de quelques lignes à la page 96, incitent à croire que l'on peut penser à un objet par l'intermédiaire non pas d'une propriété (ou de propriétés), ou d'une représentation, mais par le biais d'une relation dont les relata peuvent varier. Les indexicaux sont des termes référentiels et réfèrent à un objet qui rend vraie l'énonciation d'une phrase indexicale. Mon énonciation de « Je suis malade » est vraie si et seulement si le référent de «je » a la propriété d'être malade. Puisque je suis le seul objet qui puisse rendre vraie cette énonciation, je fais partie de ses conditions de vérité. Pour cette raison, les indexicaux, comme les noms, sont des termes directement référentiels. Le référent d'une énonciation de «je » est donné par la signification linguistique « le locuteur de l'énonciation ", laquelle va donner des objets qui ont la relation "être locuteur de » à une énonciation, et donc désigner différents référents pour différentes énonciations. En ce sens, les indexicaux se distinguent des noms propres, qui réfèrent sans profil descriptif.

Qu'est-ce que le locuteur de «Je» a à l'esprit lorsqu'il dit «je»? Comment pense-t-il à lui-même ? La signification linguistique du terme fait que l'énonciation ne peut qu'être à propos de lui-même. Toutefois, sa signification linguistique ne peut constituer une représentation de lui-même au sens psychologique fort. Le locuteur pense à lui-même au moyen d'une relation et d'un événement, d'une énonciation, et non d'une représentation. La relation à un événement ne peut être réduite à une représentation, puisque l'énonciation n'est pas une représentation dans une interprétation minimale de cette notion. Si l'on écarte la signification, on a peine à imaginer ce que serait la représentation du locuteur qui fait que, en vertu de cette représentation, il pense forcément à lui-même. Si le locuteur avait une représentation de lui-même, il serait aussi possible qu'il ait une mauvaise représentation de lui-même et que, croyant penser à lui-même, il pense en fait à un autre objet. Cette possibilité n'est pas plausible à cause du comportement sémantique de «je ». Qui plus est, « je » ne donne aucun indice sur la manière dont l'individu pense à lui-même - ne donne aucun accès à ce qui serait un état mental de ce locuteur qui représenterait ce seul locuteur. Il est aussi peu plausible que le terme « je » soit une représentation du locuteur. Notons que cela n'implique pas qu'il n'existe pas d'état mental caractéristique chez un locuteur qui dit "Je suis malade. » Mais rien n'incite à penser qu'il s'agit d'une représentation de soi. On peut donc émettre des doutes quant à la thèse selon laquelle si on pense à un objet, alors on a une représentation de cet objet. Enfin, si l'intentionnalité est cette capacité de représenter un objet qui peut ne pas exister, et si on ne peut penser à soi si on n'existe pas, alors penser à soi ne fait pas appel à cette capacité. Penser à soi ne résulte pas de l'intentionnalité du mental. 
L'objet auquel réfère mon énonciation de «je » dans «Je suis philosophe » fait partie des conditions de vérité de l'énonciation, soit une proposition singulière, mais celle-ci ne peut constituer le contenu de pensée que j'avais au moment de l'énonciation. Vous auriez pu exprimer la même proposition singulière en disant «Tu es philosophe. » Vous n'auriez alors certainement pas exprimé la même pensée. En ce sens, si les propositions singulières ont un rôle important dans l'analyse de la pensée humaine, elles le font en obligeant à distinguer clairement ce que Frege identifiait, soit le contenu de pensée et les conditions de vérité. Elles réussissent à convaincre que l'analyse du contenu de la pensée humaine sur la base des conditions de vérité des énonciations n'est pas aussi directe et fructueuse qu'on l'espérait.

Supposons maintenant que vous vouliez rapporter ce que je pense. Vous diriez probablement « Richard pense qu'il est philosophe» ou « Richard se pense philosophe ». Mais le «il » de la première phrase ne peut être le «il » coréférentiel bien connu. Castaneda ${ }^{8}$ dirait que c'est un quasi-indicateur. C'est une façon de nommer le problème, et non une solution. La phrase en contexte d'attitude propositionnelle ne donne aucun indice quant à la forme ou au contenu de la pensée du locuteur. Il serait tentant d'endosser la stratégie des modes de présentation pour analyser les propositions exprimées par des phrases comme "Richard croit qu'il est philosophe ». Mais rappelons que le locuteur disant " je » parle de lui-même en vertu de sa relation à l'énonciation, et non d'une représentation de lui-même.

Si je veux parler de moi, je peux utiliser « je ». J'établirai alors une relation à un objet sans clairement recourir à une représentation mentale de cet objet; si je veux parler de Stumpf, je peux utiliser "Stumpf». J'hésite à affirmer que le nom propre soit une représentation au sens où l'entend Brentano: ce n'est certainement pas mental. De plus, si j'ai une représentation mentale, quoi que cela veuille dire, ma représentation est certainement trop peu spécifique et ne se dirige pas vers lui et uniquement vers lui. Par exemple, on ne peut exclure que ma représentation soit aussi une représentation du double de Stumpf. Un locuteur utilisant «je » ou un nom propre pense-t-il à quelque chose? En un sens, oui. Mais sa représentation de cette chose, si représentation il y a, est effectivement opaque, sinon obscure. Il est douteux que, pour parler de quelque chose, ou pour penser à quelque chose, il soit toujours nécessaire d'avoir une représentation mentale de cette chose. Parfois, des ressources linguistiques suffisent.

8. H.-N. Castaneda, «» Indicators and Quasi Indicators», American Philosophical Quarterly 4, 1967, 85-100. 